\title{
Fabrication of ultrasmall magnets by electroplating
}

\author{
W. Xu, J. Wong, C. C. Cheng, R. Johnson, and A. Scherer \\ California Institute of Technology, Pasadena, California 91125
}

(Received 2 June 1992; accepted 15 September 1995)

\begin{abstract}
We use high voltage electron beam lithography followed by electroplating to define small metal features on semiconductor substrates. These have been used to form high resolution etch masks, dense nanomagnet arrays, and highly anisotropic metal nanostructures. To reproducibly obtain uniform arrays of such structures, we have developed an end-point detection technique, which is based on in situ observation of the electrodeposition process. (c) 1995 American Vacuum Society.
\end{abstract}

\section{INTRODUCTION}

The ultimate density of magnetic storage media depends on the minimum spacing between magnetic domains, their quality, and the interaction between adjacent magnets. ${ }^{1}$ As the storage density is increased, new methods for reading the magnetic field with high spatial resolution have to be developed. Miniaturization of magnetic storage media can now be explored through the use of high resolution microfabrication techniques such as high voltage electron beam lithography which allows us to routinely generate masks in resist material with lateral dimensions of $50 \mathrm{~nm}$ and below. ${ }^{2}$ For the fabrication of magnet arrays with in-plane magnetic shape anisotropy, we can use a lift-off process in which the magnetic material is vapor deposited through a line mask. The width and length of the magnets are determined lithographically, and the thickness of the magnets is controlled during the magnet deposition process. However, for the fabrication of small magnets with shape anisotropy perpendicular to the substrate surface, the use of metal lift-off techniques is limited since the organic resist layer must be substantially thicker than the vapor deposited magnetic layer.

The problem of obtaining high aspect ratio perpendicularly anisotropic nanomagnets has been solved in the past by electroplating magnetic metals to create vertical magnetic pillars. ${ }^{3}$ The smallest size and shape of the resulting nanomagnets are ultimately determined by the interaction of the electron beam with the resist layer by forward and backscattered electrons (proximity effects). For conventional electron beam exposure energies, forward scattering of electrons in the resist and proximity effects limit the aspect ratio and ultimate density of magnet arrays. To improve this anisotropy, we can either use a trilevel resist technique or high voltage electron beam lithography on a single resist microplating mask. Here we demonstrate the benefits of combining high energy electron beam lithography with metal electroplating and optical end-point detection as a powerful method for fabricating arrays of very small magnets.

\section{PROCEDURE}

The procedure used for electrodeposition of nickel is schematically summarized in Fig. 1. First, we prepare our semiconducting or insulating substrate by vapor depositing a thin conducting gold layer in a pattern, which electrically connects a beam-writing pad with a probe contact pad. We then spin on a single high molecular weight polymethyl- methacrylate (950K PMMA) resist layer. This resist is then exposed by using a Philips EM-430 scanning electron transmission microscope (STEM) with an acceleration voltage of $250 \mathrm{kV}^{2}$ Following the lithographic exposure, the beamwritten patterns are developed in a 3:7 cellusolve-methanol mixture. We then electrodeposit nickel, either from a nickel sulfonate or a nickel sulfate electrolyte, into the holes formed in the PMMA resist. Electroplating is done under a probe station without agitation, which allows us to optically observe the deposition process. The cathode contact is made by using a photoresist-coated probe, whereas the anode consists of an annular nickel cup with an opening cut in the center. Depending on the electroplating time, we either obtain high aspect ratio nickel pillars or mushroomlike structures, as shown in Fig. 1. Although the individual magnets are much smaller than the wavelength of light, a transition between these two geometries gives rise to an abrupt change in the reflectivity from the magnet array surface and can immediately be detected under the microscope.

Finally, when the electroplating process is complete, the PMMA can be removed by an oxygen plasma etch, which reveals the magnetic nanostructures. This procedure is not

\section{Electron beam lithography}

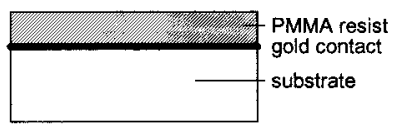

2. Pattern development
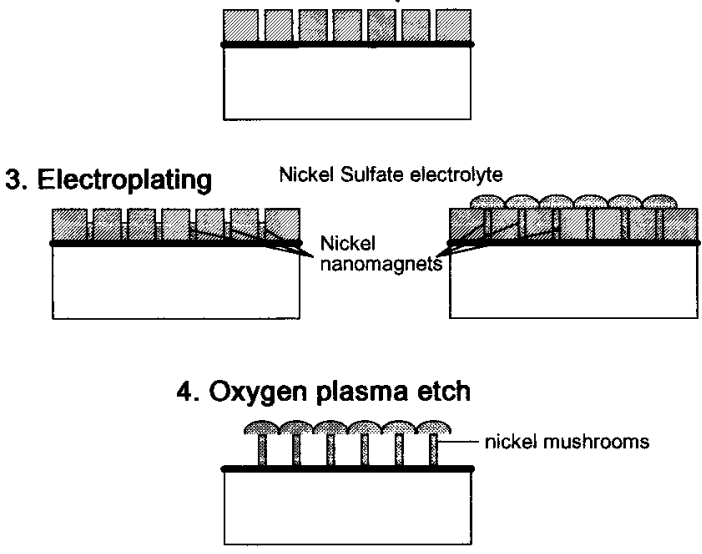

FIG. 1. Schematic of the fabrication procedure used to generate ultrasmall magnets. 
necessary or desirable unless the magnetic arrays are to be subsequently measured by electron microscopy, since the PMMA serves as an excellent matrix providing mechanical and chemical stability to the magnets. To examine the magnetic nanostructures, we use scanning electron microscopy (SEM) and reflection electron microscopy (REM) ${ }^{4}$ in the Philips EM-430 STEM. We use these techniques to measure the size, the anisotropy, the surface quality, and the crystallinity of the nickel nanostructures. The magnetic quality of the structures is also measured using a magnetic force microscope (MFM). ${ }^{5}$

\section{RESULTS AND DISCUSSION}

\section{A. Characterization of uniform magnet arrays}

After electrodeposition, scanning electron microscopy allows us to image only the tops of the magnets [Fig. 2(a)]. To reveal the complete magnetic structure, the PMMA can be removed with an oxygen plasma etch (Fig. 3). Alternatively, the magnets can be imaged with high voltage $(100 \mathrm{kV})$ scan-

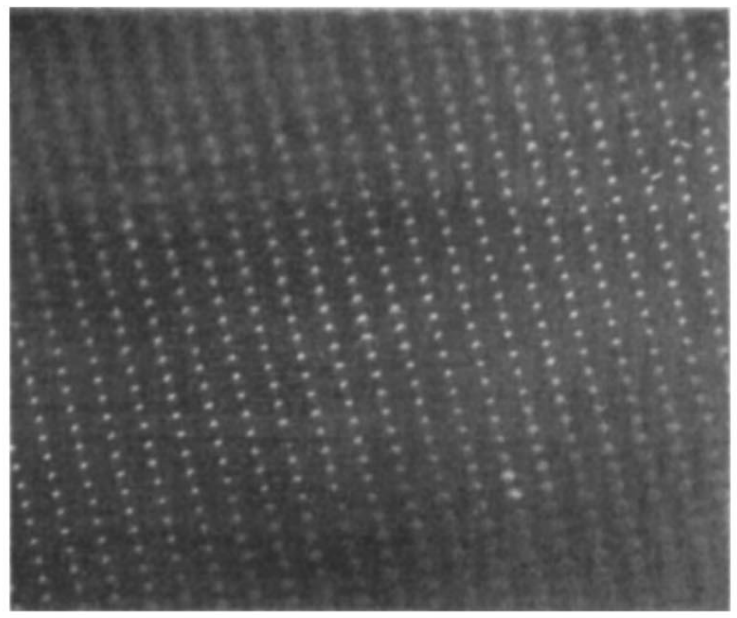

(a)

\section{$\overline{1 \mu m}$}

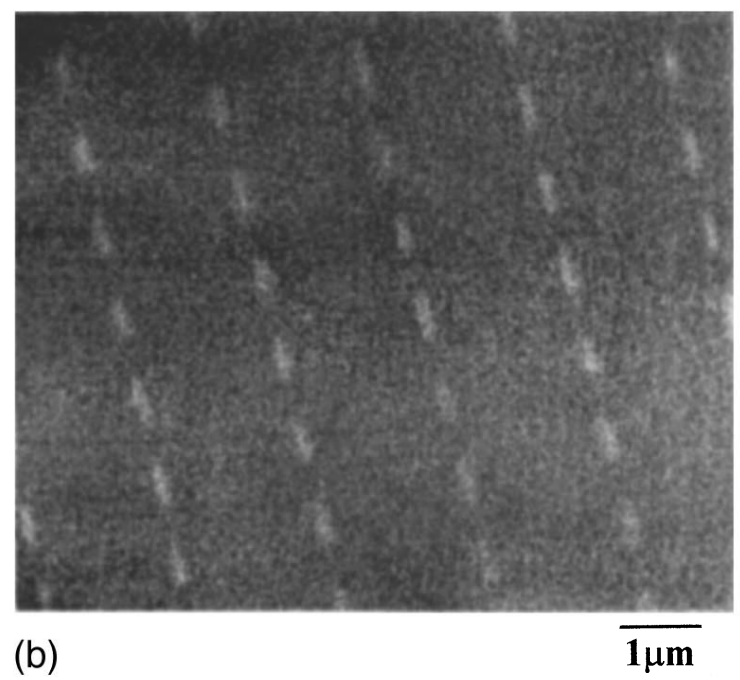

FIG. 2. (a) SEM micrograph of a nickel magnet array before removal of the PMMA resist. (b) High-voltage backscattered electron micrograph showing the nickel posts underneath the PMMA layer.
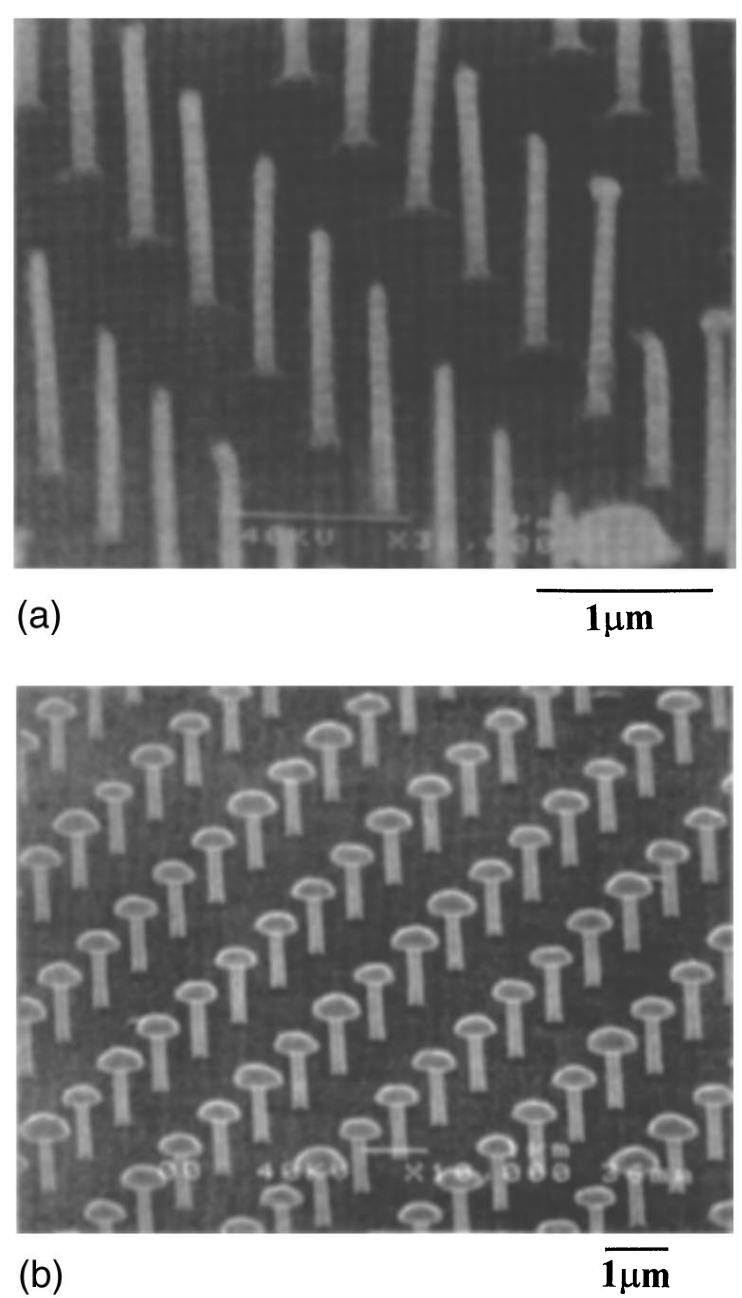

FIG. 3. (a) SEM micrograph of the array after oxygen plasma etching to remove the PMMA. (b) SEM micrograph of an overplated micromagnet array showing the mushroom shape characteristic of isotropic metal deposition.

ning electron microscopy using the backscattered electron detector [shown in Fig. 2(b)]. Since the electron beam resist serves as a mold into which the magnetic material is deposited, it is necessary to determine the time at which the electrodeposited metal completely fills the holes in the resist to prevent isotropically plated metal from forming on top of the magnet [Fig. 3(b)]. As in macroscopic plating processes, the electrodeposition rate is dependent on the current density, the nickel concentration in the electrolyte, the temperature, and the agitation of the solution. ${ }^{6}$ We find that, in lithographic samples, where extremely small areas are electrodeposited, it is very difficult to precisely predict the surface area to be plated, and therefore no reproducible value for the current density is obtained. Moreover, the deposition rate is also found to depend on the size of the feature that is to be plated.

\section{B. In situ observation and end-point detection}

As the deposition rate cannot be accurately determined $a$ priori, it is necessary to develop an in situ end-point detection system that can be used to determine the optimum electroplating time. By measuring the height and the width of 


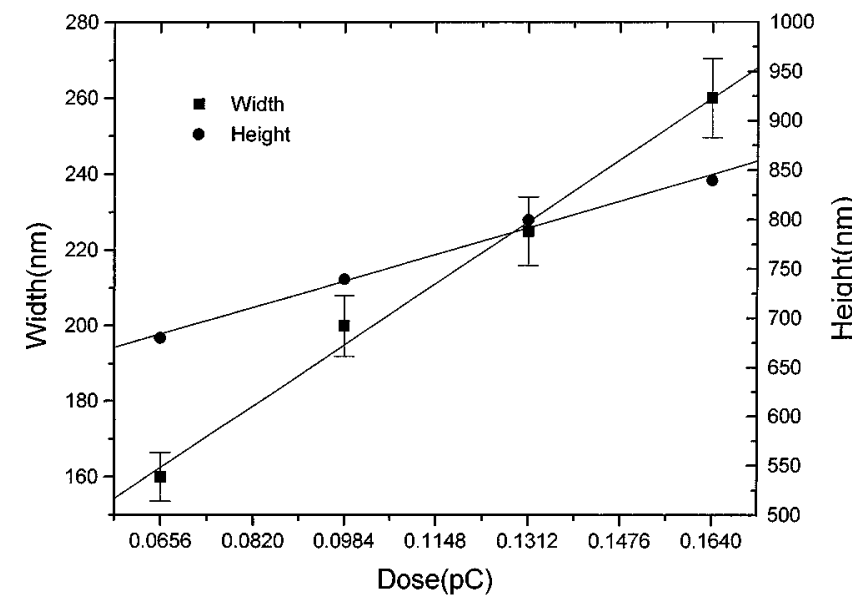

FIG. 4. Measured dependence of the height and width of nanomagnets on the exposure dose (in $\mu \mathrm{C}$ ).

electroplated magnets and relating these to the exposure dose, we have found a systematic relationship between the deposition rate and the size of the nanostructure to be plated (Fig. 4). We find that the electroplating rate is slightly faster in larger holes than in small structures, and therefore it is possible to use arrays of larger $(150 \mathrm{~nm})$ magnets as sacrificial internal standards to stop the electrodeposition process when these test regions are filled. As the plated nanostructures change from pillars to mushrooms, we observe a significant change of contrast in the probe station. Since a series of different sized magnet arrays are usually defined for optimization of the lithographic dose, this end-point detection is a convenient method for reproducibly obtaining highly anisotropic magnets [Figs. 3(a) and 3(b)].

\section{Electroplated etch masks}

Since nickel is an excellent etch mask, we have used the electroplated nickel posts to transfer anisotropic structures into the underlying semiconductor. The large thickness of the nickel makes mask electroplating a convenient method for defining very robust dry etch masks without the use of complex multilayer contrast enhancement schemes. We demonstrate this technique in Fig. 5, where we show plated nickel pillars that were used to etch to a $1.5 \mu \mathrm{m}$ depth in a silicon

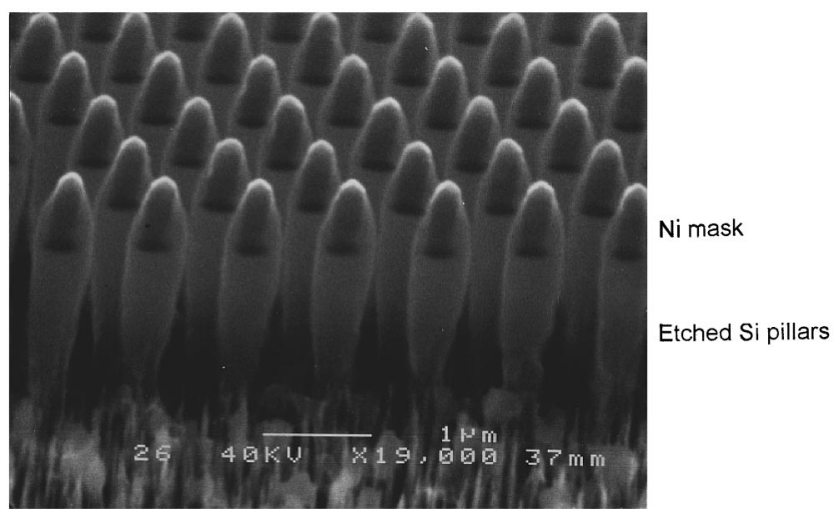

FIG. 5. Etched silicon pillars defined by using a $1-\mu \mathrm{m}$-thick electroplated nickel etch mask.

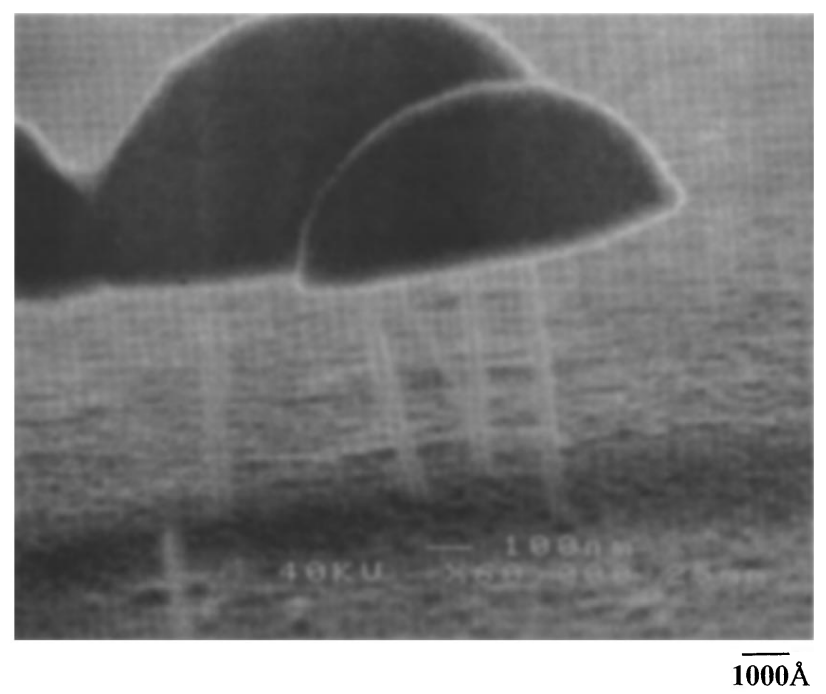

FIG. 6. Overplated 30-nm-wide magnetic columns showing the resolution of the electroplating process.

substrate by using a $\mathrm{CCl}_{2} \mathrm{~F}_{2} / \mathrm{NF}_{3}$ reactive ion etch. During the etching process, the thin vapor deposited gold membrane onto which the mask was plated was removed almost immediately, leaving the substrate to be etched as deep as is desired. For the deposition of similar thicknesses of metal mask, significantly thinner resist layers can be used when electroplating than when using an evaporation/lift-off process. This can result in correspondingly higher pattern resolution and/or etch anisotropy.

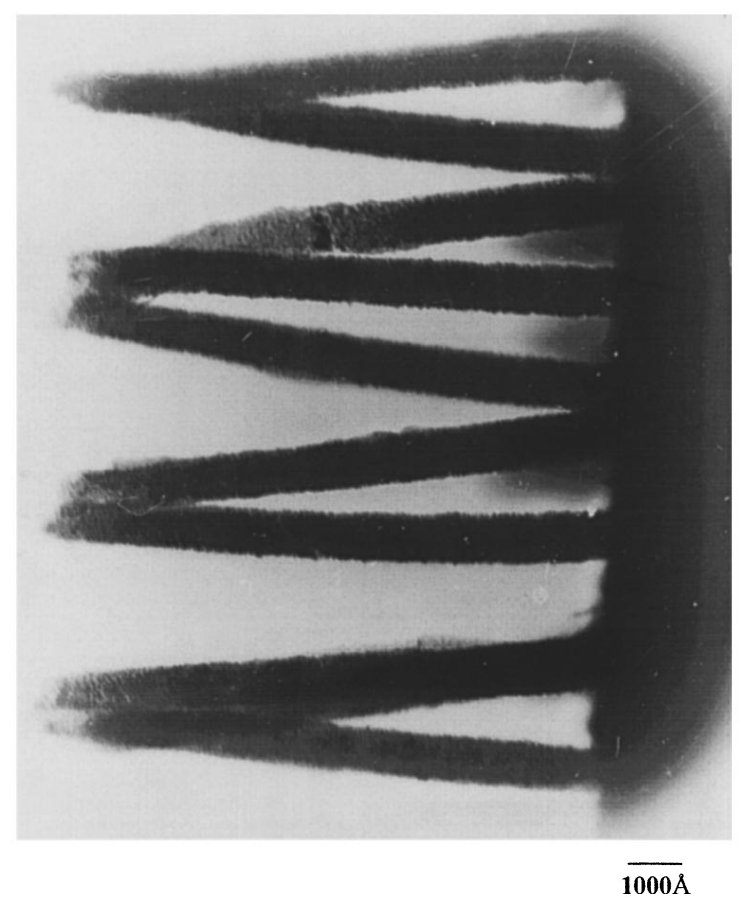

FIG. 7. Reflection electron micrograph of 100-nm-wide and 1 - $\mu$ m-tall magnets. 


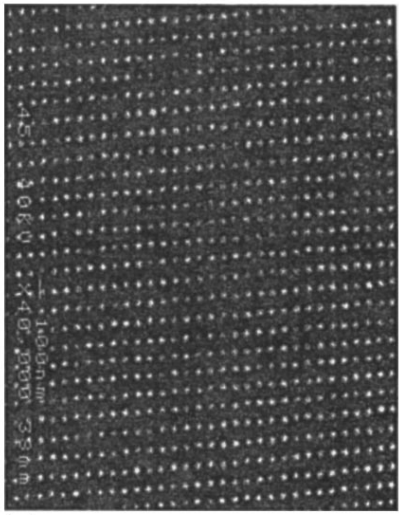

(a)

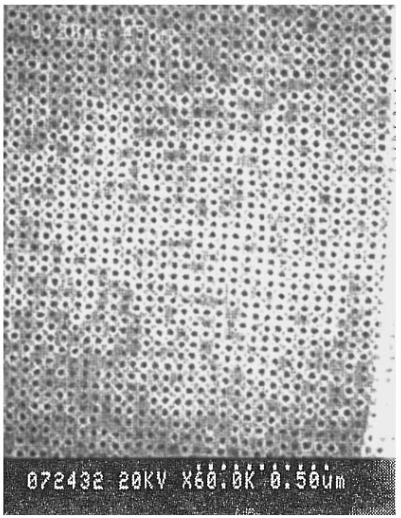

(b)

FIG. 8. (a) High density $\left(>65 \mathrm{Gbit} / \mathrm{in}^{2}{ }^{2}\right)$ magnetic recording media. Nanomagnet arrays of $20 \mathrm{~nm}$ Ni pillars with $100 \mathrm{~nm}$ spacing were microfabricated. (b) SEM micrograph of $12 \mathrm{~nm}$ holes etched into GaAs with $45 \mathrm{~nm}$ spacing.

\section{Ultrasmall magnets, mushroom structures, and reflection microscopy}

Arrays of uniform nanomagnets as small as $20 \mathrm{~nm}$ in diameter have been produced and were examined through scanning electron microscopy and reflection electron microscopy. The current density during electroplating is very difficult to determine, and the most common failure mode of this process lies in incorrect timing of the electrodeposition which results in plating of mushroomlike structures. In Fig. 6 , we show a 30-nm-wide and 0.4- $\mu \mathrm{m}$-tall lithographic column after such an electroplating and resist removal. Plated magnets were also examined by reflection electron microscopy, a technique that allows us to obtain a dark-field transmission electron micrograph from the sample surface and image the crystalline structure of the pillars. From the reflection electron micrograph of Fig. 7 we observe a microcrystalline plating morphology after nickel sulfonate deposition of 100-nm-wide magnetic pillars. Other REM images and diffraction patterns confirm that the nickel crystallite size is approximately $5 \mathrm{~nm}$. Electroplating is also an excellent method for determining the shape of the electron beam interaction volume in the electron beam resist. When we compare the sidewall angle of magnets defined through electron beam lithography at 30 and at $250 \mathrm{kV}$, we observe a significantly lower anisotropy as a result of forward scattering of the incident electron beam during low-voltage exposure, ${ }^{7}$ which results in magnets with shapes resembling truncated cones. At higher electron beam energies, it is possible to define magnets with vertical sidewalls. Preliminary measurements using a MFM indicate that the resulting anisotropic nickel pillars are indeed very good magnets.

\section{E. Dense magnetic arrays for storage media}

The inherently high contrast of high voltage electron beam lithography also allows the fabrication of very dense arrays of small structures. Since the density of magnetic particles ultimately limits the memory storage capacity, it is desirable to explore the highest possible density of magnetic structures in regular arrays. We have used e-beam lithography to define arrays of $30 \mathrm{~nm}$ magnets with $80 \mathrm{~nm}$ pitch [Fig. 8(a)]. This packing density translates into an equivalent memory storage capacity of over $60 \mathrm{Gbit} / \mathrm{in}^{2}{ }^{2}$ The density of the magnets can be further increased by optimizing the electron beam lithography parameters. Under optimal conditions, we define $12 \mathrm{~nm}$ holes in 100-nm-thick PMMA resist with $45 \mathrm{~nm}$ center spacings [Fig. 8(b)]. We presently use this fabrication capability to determine the limits of magnetic storage, the interaction between magnets, and the problems associated with reading the magnetic orientation of individual magnets in such dense arrays.

\section{CONCLUSIONS}

High resolution electron beam lithography, together with careful electrodeposition, allows us to define highly anisotropic magnets with lateral sizes below $30 \mathrm{~nm}$. Magnets with aspect ratios above 15:1 have been demonstrated by using high voltage electron beams, which minimize the electron scattering in the electron beam resist. In addition, very dense patterns with spacings below $100 \mathrm{~nm}$ have been constructed, with correspondingly large storage densities for magnetic media. End-point detection based on the electrodeposition rate dependence on lithographic structure width allows us good plating reproducibility even for dense arrays of the smallest magnets.

\section{ACKNOWLEDGMENTS}

The authors wish to acknowledge many helpful discussions with S. Heckman at Hughes Research Laboratory and Dr. S. Schultz and R. O'Barr from the Center for Magnetic Recording Research (CMRR) at the University of California, San Diego. This work was supported in part by NSF Grant No. ECS93-10681.

${ }^{1}$ T. Ohkubo, J. Kishigami, K. Yanagisawa, and R. Kaneko, IEEE Trans. Magn. 27, 5286 (1991).

${ }^{2}$ A. Scherer and H. G. Craighead, Appl. Phys. Lett. 49, 1284 (1986); R. E. Behringer, P. M. Mankiewich, and R. E. Howard, J. Vac. Sci. Technol. B 5, 326 (1987); S. Mackie and S. P. Beaumont, Solid State Technol. August, 117 (1985); A. N. Broers, IBM J. Res. Dev. 3, 502 (1988).

${ }^{3}$ P. R. Krauss, P. B. Fischer, and S. Y. Chou, J. Vac. Sci. Technol. B 12, 3639 (1994); S. Y. Chou, M. S. Wei, P. R. Krauss, and P. B. Fischer, J. Appl. Phys. 76, 6673 (1994); L. Piraux, J. M. George, F. F. Despres, C. Leroy, E. Ferain, R. Legras, K. Ounadjela, and A. Fert, Appl. Phys. Lett. 75, 2484 (1994)

${ }^{4}$ B. P. Van der Gaag and A. Scherer, Appl. Phys. Lett. 56, 481 (1990).

${ }^{5}$ G. A. Gibson, J. F. Smyth, and S. Schultz, IEEE Trans. Magn. 27, 5187 (1991).

${ }^{6}$ V. L. Lainer and N. T. Kudryatsev, Fundamentals of Electroplating 2, 3rd ed. (U.S. Department of Commerce, New York, 1957), p. 192.

${ }^{7}$ D. E. Newbury and R. L. Myklebust, Ultramicroscopy 3, 391 (1979); D. F. Kyser, J. Vac. Sci. Technol. B 1, 1391 (1983); D. F. Kyser and K. Murata, IBM J. Res. Dev. July 352 (1974). 